\title{
ON VALUES OF EXPONENTIAL SUMS
}

\section{CHUNGMING AN}

ABSTRACT. An exponential sum is defined by

$$
G(F, \varphi, \alpha)=\sum_{\gamma \in(Z / q Z)^{n}} \exp (2 \pi i(\varphi F(\gamma)+\langle\alpha, \gamma\rangle))
$$

for $\varphi=a / q \in Q, a \in R^{n}$, and a positive-definite form $F(x)$ in $n$ variables of degree $\delta$. Its value is studied and the definition is extended to an irrational $\varphi$.

1. A zeta function associated with the positive-definite forms was studied in [1], [2]. Its residue at the only possible simple pole is given in an explicit form which is closely related to the exponential sums of certain type. These sums can alșo be viewed as a generalization of the Gaussian sum.

2. Let $Z, Q$ and $R$ be the sets of integers, rationals and reals, respectively. $F(x)=F\left(x_{1}, \ldots, x_{n}\right)$ is a positive-definite form in $n$ variables of degree $\delta$, i.e. $F(x)=0$ iff $x=0$. For $\varphi=a / q \in Q$ and $\alpha \in R^{n}$, we consider the infinite series

$$
\zeta(F, \varphi, a, s)=\sum_{\gamma \in Z^{n}-\{0\}} F(\gamma)^{-s} e(\varphi F(\gamma)+\langle a, \gamma\rangle),
$$

$s=\sigma+t i$ and $\operatorname{Re}(s)>n / \delta=\sigma_{0}$, and the sums

$$
G_{1}(F, \varphi, \alpha)=q^{-n} \sum_{\eta \in(Z / q Z)^{n}} e^{(\varphi F(\eta)+\langle\alpha, \eta\rangle),}
$$

and for the smallest positive integer $m$ such that $m, \in Z$ and $m a \in Z^{n}$,

$$
G_{2}(F, \varphi, \alpha)=m^{-n} \sum_{\eta \in(Z / m Z)^{n}} e(\varphi F(\eta)+(\alpha, \eta))
$$

where $e(c)=\exp (2 \pi i c)$ for a real number c. Clearly, $G_{1}=G_{2}$ if $m=q$.

The following theorem is proved in [1].

Theorem. For a $\in Q^{n}, \zeta(F, \varphi, a, s)$ has a meromorphic continuation into the whole s-plane and the residue at its only possible simple pole $s=\sigma_{0}=$ $n / \delta$ is

$$
\operatorname{Res}(\zeta)=(2 \pi)^{\sigma}{ }^{0} \Gamma\left(\sigma_{0}\right)^{-1} G_{2}(F, \varphi, \alpha) \int_{R^{n}} \exp (-2 \pi F(x)) d x
$$

We see in this theorem that $\alpha$ is restricted to have rational coordinates. The case $\alpha \in R^{n}$ and $\varphi=0$ was studied in [2]. In this paper we shall obtain the general result. 
Theorem. For $\boldsymbol{\varphi}=a / q$ and $\alpha \in R^{n}, \zeta(F, \varphi, \alpha, s)$ is an entire function unless $q \alpha \in Z^{n}$. In case $q \alpha \in Z^{n}$, the function $\zeta(F, \varphi, \alpha, s)$ has only one possible simple pole at $s=\sigma_{0}$ with the residue

$$
\operatorname{Res}(\zeta)=(2 \pi)^{\sigma}{ }^{0} \Gamma\left(\sigma_{0}\right)^{-1} G_{1}(F, \varphi, \alpha) \int_{R^{n}} \exp (-2 \pi F(x)) d x
$$

Proof. We put

$$
\xi(F, \varphi, \alpha, s)=(2 \pi)^{-s} \Gamma(s) \zeta(F, \varphi, \alpha, s) .
$$

By a Mellin transform, we get the integral representation, when $\sigma>\sigma_{0}$,

$$
\begin{aligned}
\xi(F, \varphi, \alpha, s) & =\int_{0}^{\infty}\left[\sum_{\gamma \in Z^{n}-\{0\}} e((\varphi+t i) F(\gamma)+\langle\alpha, \gamma\rangle)\right] t^{s-1} d t \\
& =I_{1}(s)+I(s)
\end{aligned}
$$

where

$$
\begin{aligned}
I_{1}(s) & =\int_{0}^{1}\left[\sum_{\gamma \in Z^{n}-\{0\}} e((\varphi+t i) F(\gamma)+\langle\alpha, \gamma\rangle)\right] t^{s-1} d t \\
I(s) & =\int_{1}^{\infty}\left[\sum_{\gamma \in Z^{n}-\{0\}} e((\varphi+t i) F(\gamma)+\langle\alpha, \gamma\rangle)\right] t^{s-1} d t .
\end{aligned}
$$

It is easy to show that $I(s)$ represents an entire function (see [11). If we put

then $I_{1}(s)=I_{2}(s)-1 / s$.

$$
I_{2}(s)=\int_{0}^{1}\left[\sum_{\gamma \in Z^{n}} e((\varphi+t i) F(\gamma)+\langle\alpha, \gamma\rangle)\right] t^{s-1} d t
$$

For $\gamma \in Z^{n}$, we write $\gamma=\beta+q \eta$, where $\beta=\left(\beta_{1}, \ldots, \beta_{n}\right) Z^{n}, 0 \leq \beta_{l}$ $\leq q$ and $\eta \in Z^{n}$. Then $F(\gamma)=F(\beta)+M q$, M being an integer which depends only on $\beta$ and $\eta$. Hence

$$
e((\boldsymbol{\varphi}+t i) F(\gamma)+\langle\alpha, \gamma\rangle)=e(\boldsymbol{\varphi} F(\beta)+\langle\alpha, \beta\rangle) e(t i F(\beta+q \eta)+\langle\alpha, q \eta\rangle) .
$$

So

$$
I_{2}(s)=\int_{0}^{1}\left[\sum_{\beta \in(Z / q Z)^{n} ; \eta \in Z^{n}} e(\varphi F(\beta)+\langle\alpha, \beta\rangle) e(t i F(\beta+q \eta)+\langle\alpha, q \eta\rangle)\right] t s^{-1} d t .
$$

By applying the Poisson summation formula

for

$$
\sum_{\eta \in Z^{n}} \phi(-x+\eta)=\sum_{\eta \in Z^{n}} \hat{\phi}(\eta) e(\langle x, \eta\rangle)
$$

$$
\phi(x)=\exp (-2 \pi t F(\beta+q x)) \text { and } \hat{\phi}(y)=\int_{\mathbf{R}^{n}} \phi(x) e(-\langle x, y\rangle) d x \text {, }
$$

along with the change of variables $\beta+q x \rightarrow t^{-1 / \delta} x$, we obtain 


$$
\begin{aligned}
I_{2}(s)=\int_{0}^{1}\left[q^{-n} \sum_{\beta \in(Z / q Z)^{n}} e(\varphi F(\beta))\right. & \sum_{\eta \in Z^{n}} e\left(\left\langle\beta, q^{-1} \eta\right\rangle\right) \\
& \left.\cdot \hat{\psi}\left(q^{-1} t^{-1 / \delta}(-q \alpha+\eta)\right)\right] t^{s-\sigma_{0}-1} d t
\end{aligned}
$$

where $\psi(x)=\exp (-2 \pi F(x))$ is a Schwartz function and so is $\hat{\psi}(x)$.

The proof is divided into two cases.

Case (a). If $q \alpha-\eta \neq 0 \forall \eta \in Z^{n}$, then

$$
\left|\hat{\psi}\left(q^{-1} t^{-1 / \delta}(-q \alpha+\eta)\right)\right| \leq C q^{2 \cdot v} t^{2 v / \delta}|\eta-q \alpha|^{-2 N}
$$

where $N$ is a positive integer and $C$ is a positive constant independent of $t$ and $\eta$. Thus the integral form of $I_{2}(s)$ is majorized by the series

$$
\frac{C q^{2 . v}}{s+(2 N-n) / \delta} \sum_{\eta \in Z^{n} ; \eta \neq q \alpha}|\eta-q \alpha|^{-2 . N}
$$

which converges for $N>n / 2$. Hence $I_{2}(s)$ represents an entire function.

Case (b). If $q \alpha \in Z^{n}$, then

$$
\begin{aligned}
I_{2}(s) & =I_{3}(s)+\int_{0}^{1}\left(q^{-n} \sum_{\beta \in(Z / q Z)^{n}} e(\varphi F(\beta)+\langle\alpha, \beta\rangle) \hat{\psi}(0)\right) t^{s-\sigma_{0}-1} d t \\
& =I_{3}(s)+\frac{\hat{\psi}(0)}{s-\sigma_{0}} G_{1}(F, \varphi, \alpha),
\end{aligned}
$$

where

$$
\begin{aligned}
I_{3}(s)=\int_{0}^{1}\left[q^{-n} \sum_{\beta \in(Z / q Z)^{n}} e(\varphi F(\beta))\right. & \sum_{\eta \neq q a} e\left(\left\langle\beta, q^{-1} \eta\right\rangle\right) \\
& \left.\cdot \hat{\psi}\left(t^{-1 / \delta} q^{-1}(-q \alpha+\eta)\right)\right] t^{s-\sigma_{0}-1} d t .
\end{aligned}
$$

By a method similar to that of Case (a), we see that $I_{3}(s)$ is entire.

We have proved that

$$
\xi(F, \rho, \alpha, s)=I(s)+I_{3}(s)-1 / s+\frac{K(\alpha)}{s-\sigma_{0}} G_{1}(F, \varphi, \alpha) \int_{R^{n}} \exp (-2 \pi F(x)) d x
$$

where $I(s)$ and $I_{3}(s)$ are entire, $K(\alpha)=1$ if $q \alpha \in Z^{n}$ and $=0$ otherwise.

$\xi(F, \varphi, a, s)$ has a simple pole at $s=0$ with residue $=-1$ regardless of $F(x), \varphi$ and $a$. But the point $s=0$ is a removable singularity of the function $\zeta(F, \varphi, \alpha, s)$.

The theorem follows immediately.

A trivial consequence following the above two theorems is

Corollary. If $\alpha \in Q^{n}$ and $q \alpha \notin Z^{n}$, then $G_{2}(F, \varphi, \alpha)=0$. 
3. From the definition of $G_{2}(F, \varphi, a)$, we shall easily see that the sum is independent of the choice of $m$, i.e. $m$ is not necessarily the smallest one. The same idea leads to

Proposition. For $:=a / q \in Q$ and $a \in Q^{n}$, let

$$
S_{N}(F, \varphi, \alpha)=N^{-n} \sum_{\eta \in(Z / N Z)^{n}} e^{(\varphi F(\eta)+\langle\alpha, \eta\rangle) .}
$$

Then

$$
\begin{aligned}
\lim _{N \rightarrow \infty} S_{N}(F, \varphi, \alpha) & =G_{1}(F, \varphi, \alpha) \quad \text { if } q \alpha \in Z^{n}, \\
& =G_{2}(F, \varphi, \alpha) \text { if } q \alpha \notin Z^{n} .
\end{aligned}
$$

Proof. We shall prove for $q \alpha \in Z^{n}$. The other case is the same. For each $N$, we put $N=k q+p, 0 \leq p<q$. then

$$
\begin{aligned}
S_{N}(F, \varphi, \alpha)= & N^{-n} \sum_{\beta(Z / k Z)^{n}} \sum_{\eta \in(Z / q Z)^{n}} e(\varphi F(\beta q+\eta)+\langle\alpha, \beta q+\eta\rangle) \\
& +\sum^{\prime} e(\varphi F(\beta q+\eta)+\langle\alpha, \beta q+\eta\rangle)
\end{aligned}
$$

where $\Sigma^{\prime}$ is the sum over $(Z / N Z)^{n}-(Z / k q Z)^{n}$, the set-theoretic complement.

Since $N \rightarrow \infty$ iff $k \rightarrow \infty$ and $\left|\Sigma^{\prime}\right| \leq C k^{n-1} q^{n}$ for a constant $C$ indepenent of $k$ and $N$, it is easy to see that

$$
\lim _{N \rightarrow \infty} N^{-n}\left|\sum^{\prime}\right| \leq \lim _{k \infty \infty} \frac{C k^{n-1} q^{n}}{(k q+p)^{n}}=0 .
$$

Again $F(\beta q+\eta)=F(\eta)+M q$ for an integer $M$. We have

$$
\begin{aligned}
\lim _{N \rightarrow \infty} S_{. V}(F, \varphi, \alpha) & =\lim _{k \rightarrow \infty}(k q+p)^{-n} \sum_{\beta \in(Z / k Z)^{n}} \sum_{\eta \in(Z / q Z)^{n}} e(\varphi F(\eta)+\langle\alpha, \eta\rangle) \\
& =\lim _{k \rightarrow \infty}(k q+p)^{-n} k^{n} \sum_{\eta \in(Z / q Z)^{n}} e(\varphi F(\eta)+\langle\alpha, \eta\rangle) \\
& =G_{1}(F, \varphi, \alpha) .
\end{aligned}
$$

Hence we may use the limit as the definition of the sums $G_{1}$ and $G_{2}$. By the proposition and the corollary, the limit is $G_{1}$ if $q \alpha \in Z^{n}$ and equals to 0 if $q a \notin Z^{n}$.

The definition by the limit also allows us to define the sum (we may call it a Gaussian sum) for any $\varphi \in R$. We put, if it exists, $G(F, \varphi, \alpha)=$ $\lim _{N \rightarrow \infty} S_{N}(F, \varphi, \alpha)$ for $\varphi \in R$ and $\alpha \in R^{n}$. When $\varphi$ is an irrational number, a theorem on the uniform distribution modulo one for polynomials [3] gives 
Proposition. $G(F, \varphi, \alpha)=0$ if $\varphi$ is an irrational number, or $\alpha$ has at least one irrational coordinate.

Thus the only sum whose value is possibly nonzero is $G_{1}(F, \varphi, a)$ when $\varphi=a / q$ and $q \alpha \in Z^{n}$.

4. Finally, we would like to make a conjecture. The zeta function $\zeta(F, \varphi, a, s)$ can also be defined for an irrational $\varphi$. Unfortunately, the method we use cannot be applied to any $\varphi$ in order to get the analytic continuation for $\zeta(F, \boldsymbol{\varphi}, \alpha, s)$. Since the residue of $\zeta(F, \boldsymbol{\varphi}, \alpha, s)$, for a rational $\boldsymbol{\varphi}$, is so closely related to $G(F, \varphi, a)$ and since $G(F, \varphi, \alpha)=0$ for an irrational $\varphi$, it should be a reasonable guess that $\zeta(F, \varphi, \alpha, s)$ would be an entire function for an irrational number $\varphi$.

\section{REFERENCES}

1. Chungming $A_{n}, O_{n}$ the analytic continuation of a certain Dirichlet series, J. Number Theory 6 (1974). (1974).

2. - A generalization of Epstein's zeta function, Michigan Math. J. 21

3. J. D. van der Corput, Uniform distribution for polynomials, Acta. Math. 56 (1931), 373-456.

DEPARTMENT OF MATHEMATICS, SETON HALL UNIVERSITY, SOUTH ORANGE, NEW JERSEY 17079 